\title{
Cardiopulmonary bypass for bilateral sequential lung transplantation in patients with chronic obstructive pulmonary disease without adverse effect on lung function or clinical outcome
}

\author{
Wilson Y. Szeto, MD \\ Daniel Kreisel, MD \\ Giorgos C. Karakousis, MD \\ Alberto Pochettino, MD \\ Daniel H. Sterman, $M D^{b}$ \\ Robert M. Kotloff, MD \\ Selim M. Arcasoy, MD ${ }^{\mathrm{b}}$ \\ David A. Zisman, $M D^{\mathrm{b}}$ \\ Nancy P. Blumenthal, CRNP ${ }^{b}$ \\ Robert J. Gallop, PhDc \\ Larry R. Kaiser, $\mathrm{MD}^{\mathrm{a}}$ \\ Joseph E. Bavaria, $\mathrm{MD}^{\mathrm{a}}$ \\ Bruce R. Rosengard, MD
}

From the Department of Surgery, Division of Cardiothoracic Surgery, and the Department of Medicine, Division of Pulmonary Medicine and Critical Care, ${ }^{\mathrm{b}}$ Hospital of the University of Pennsylvania, Philadelphia, Pa, and the Department of Mathematics and Applied Statistics, ${ }^{c}$ West Chester University, West Chester, Pa.

Received for publication May 9, 2001; revisions requested June 15, 2001; revisions received Oct 10, 2001; accepted for publication Oct 24, 2001.

Address for reprints: Bruce R. Rosengard, MD, Division of Cardiothoracic Surgery, Department of Surgery, Hospital of the University of Pennsylvania, 3400 Spruce St, Silverstein 6th Floor, Philadelphia, PA 19104 (E-mail: brosenga@mail.med.upenn. edu).

J Thorac Cardiovasc Surg 2002;124:241-9

Copyright () 2002 by The American Association for Thoracic Surgery

$0022-5223 / 2002 \$ 35.00+0 \quad \mathbf{1 2 / 1 / 1 2 1 3 0 3}$

doi: $10.1067 / \mathrm{mtc} .2002 .121303$
Objective: The use of cardiopulmonary bypass in lung transplantation remains controversial. Previous studies have concluded that cardiopulmonary bypass is deleterious, but these studies were confounded by the inclusion of patients with different diagnoses undergoing single- and double-lung transplantation with elective or emergency use of bypass. The goal of this study was to determine whether cardiopulmonary bypass has deleterious effects on lung function or clinical outcome by analyzing the cases of patients with a single disease entity and elective use of bypass for bilateral sequential lung transplantation.

Methods: A retrospective review of 50 patients with chronic obstructive pulmonary disease who underwent bilateral sequential lung transplantation was performed. Fourteen patients who underwent elective cardiopulmonary bypass for $218.3 \pm 75.4$ minutes were compared to 36 control patients.

Results: After the operation, the bypass and nonbypass groups were not significantly different with respect to median duration of mechanical ventilation (1 day vs 1 day, $P=.76$ ), median stay in the intensive care unit (4 days vs 4 days, $P=.44$ ), median hospital stay (15.5 days vs 16 days, $P=.74)$, mean increase in serum creatinine level $(1.4 \pm 1.9 \mathrm{mg} / \mathrm{dL}$ vs $0.9 \pm 1.0 \mathrm{mg} / \mathrm{dL}, P=.33)$, and mean ratio of $\mathrm{PaO}_{2}$ to fraction of inspired oxygen at 1 hour $(376.6 \pm 123$ vs $357.0 \pm 218, P=.75)$, at 24 hours (309.9 \pm 92 vs $350.6 \pm 122, P=.26)$, and at 48 hours $(335.0 \pm 144$ vs $316.2 \pm 120, P=.64)$. Late outcome markers compared between the bypass and nonbypass groups were the following: 1 -year percentage predicted forced expiratory volume in 1 second $(76.1 \% \pm 17.0 \%$ vs $85.3 \% \pm 21.7 \%, P=.24)$, 30-day mortality (7.1\% vs $8.3 \%, P>.999)$, 1 -year survival $(85.7 \%$ vs $80.1 \%, P=.66$ ), 3 -year survival $(64.3 \%$ vs $58.3 \%, P=.70)$, and the prevalence of bronchiolitis obliterans syndrome ( $0 \%$ vs $36.1 \%, P=.01)$.

Conclusion: Cardiopulmonary bypass appears to have no deleterious effect on early lung function or clinical outcome. We hope that this pilot study removes some of the unwarranted fear of the use of bypass in lung transplantation for chronic obstructive pulmonary disease. 


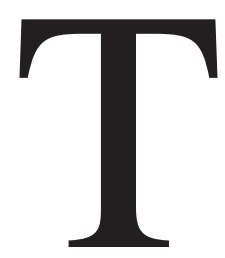

he technique of bilateral sequential lung transplantation has made the procedure of double-lung replacement possible without the use of cardiopulmonary bypass (CPB). ${ }^{1}$ However, whether to use CPB in lung transplantation remains controversial. Advocates of $\mathrm{CPB}$ argue that if $\mathrm{CPB}$ is not routinely used in the setting of bilateral sequential lung transplantation, the first implanted allograft is subjected to increased ischemia-reperfusion injury during the implantation of the second allograft. This is believed to result from the fact that the entire cardiac output is diverted to the first implanted allograft, resulting in increased blood flow and pressure. This hyperperfusion injury is believed to exacerbate the obligate ischemia-reperfusion injury consequent to transplantation. ${ }^{2-4}$ Clinically, this may manifest as acute lung injury and allograft dysfunction. Furthermore, advocates of CPB argue that the use of CPB in lung transplantation ensures stable recipient hemodynamics and facilitates difficult recipient dissection. However, the deleterious effects of CPB have been well documented since its introduction into clinical cardiac surgery. The proinflammatory effect appears to be the result of the activation of cytokines, leukocytes, and the complement cascade. ${ }^{5}$ In the setting of lung transplantation, experimental ${ }^{6,7}$ and clinical data ${ }^{8,9}$ have suggested that CPB itself is potentially associated with acute lung injury and allograft dysfunction.

Previous studies have reached differing conclusions regarding the effect of CPB in clinical lung transplantation. ${ }^{8-11}$ However, these studies are confounded by the inclusion of patients for whom CPB was used on an emergency basis, single-lung recipients in whom graft function was variably affected by disease in the remaining native lung, and patients with several diagnoses that could independently affect outcome. The goal of this study was to determine whether CPB in itself has a deleterious effect on lung allograft function and clinical outcome by analyzing the cases of patients with a single disease entity undergoing bilateral sequential lung transplantation with elective use of CPB. We therefore performed a retrospective review that selected only patients with a diagnosis of chronic obstructive pulmonary disease (COPD) undergoing bilateral sequential lung transplantation with or without $\mathrm{CPB}$. We hypothesized that CPB itself has minimal deleterious effect on allograft function and clinical outcome and that previous conclusions regarding the deleterious effects of CPB have been biased by patients' underlying diagnoses, the procedure chosen, and the circumstances in which CPB was used.

\section{Methods}

\section{Patient Population}

Between November 1991 and September 2000, a total of 252 adult lung transplantations were performed at the Hospital of the University of Pennsylvania. Of these patients, 137 patients with the diagnosis of COPD related to emphysema and $\alpha_{1}$-antitrypsin deficiency underwent either single-lung $(n=85)$ or double-lung transplantation $(n=52)$. The indication for lung transplantation was severely compromised pulmonary function with a forced expiratory volume in 1 second $\left(\mathrm{FEV}_{1}\right)$ less than $20 \%$ of predicted. In the setting of single-lung transplantation, it is difficult to account for the effects of the remaining diseased, native lung on the recipient's pulmonary function and clinical outcome. Moreover, issues of differential blood flow and airflow to the pulmonary allografts make the assessment of graft function difficult to interpret. Therefore recipients undergoing bilateral sequential lung transplantation without the use of CPB $(n=36)$ were compared with recipients undergoing bilateral sequential lung transplantation with the elective use of CPB $(n=14)$. The decision to use CPB to provide controlled pulmonary reperfusion pressure and flow to the newly implanted allograft during implantation of the second allograft was made before recipient pneumonectomy. Historically, our group has been using CPB for any single- or double-lung recipient with mean pulmonary artery pressure of $40 \mathrm{~mm} \mathrm{Hg}$ or more on single-lung ventilation with maximum medical support $\left(\mathrm{PCO}_{2}\right.$ 25-30 mm Hg, inhaled nitric oxide at $40 \mathrm{ppm}$, intravenous alprostadil at the maximum tolerable dose keeping mean arterial pressure $60 \mathrm{~mm} \mathrm{Hg}$, and isoproterenol at the maximum tolerable dose keeping the heart rate 110 beats/min). According to the operative notes and anesthesia records, in no case did acute hemodynamic instability or oxygenation desaturation occur intraoperatively after the implantation and reperfusion of the first allografts, necessitating the emergency use of CPB. CPB was instituted before implantation of both lungs in 8 cases and after implantation of the first allografts in 6 cases. Two patients who did require emergency institution of $\mathrm{CPB}$ as a result of hemodynamic instability were excluded from this study, resulting in a total of 50 patients in this analysis. No other patients were excluded. One of these patients had immediate, hyperacute lung rejection, resulting in cardiopulmonary instability, cardiopulmonary arrest, and death. This patient had a positive (100\% cytotoxicity) retrospective crossmatch and had pulmonary histologic and immunohistochemical (complement and antibody deposition) evidence of hyperacute rejection. The other patient displayed significant hemodynamic instability and pulmonary hypertension during the implantation of the second allograft while on single-lung ventilation with the newly implanted first allograft.

Recipient demographic characteristics are listed in Table 1. Baseline pulmonary function tests were performed for all patients before transplantation as part of the preoperative workup. Ischemia time was defined as the interval between crossclamping of the donor aorta and reperfusion of the transplanted lungs. Ischemia times for both lungs were individually recorded.

\section{Assessment of Allograft Function}

Assessment of early allograft function was made by measuring the following markers. Requirement for mechanical ventilation was reviewed and expressed as the median duration of intubation in days. Chest radiographic reports at approximately 1, 24, and 48 hours after transplantation were obtained, since chest radiographic evidence of infiltration has been suggested as a possible clinical marker of acute lung injury. A quantitative chest radiographic infiltrate score was assigned to each individual lung on the basis of 
TABLE 1. Preoperative characteristics

\begin{tabular}{|c|c|c|c|}
\hline Characteristic & Non-CPB & CPB & $P$ value \\
\hline Age $(y$, mean $\pm S D)$ & $49.9 \pm 8.5$ & $53.8 \pm 6.1$ & .13 \\
\hline Gender ( $\%$ male) & 62.9 & 64.3 & .93 \\
\hline $\begin{array}{l}\text { Proportion of predicted forced } \\
\text { vital capacity }(\% \text {, mean } \pm \text { SD) }\end{array}$ & $57.6 \pm 18.4$ & $57.1 \pm 14.3$ & .94 \\
\hline $\begin{array}{l}\text { Proportion of predicted } \mathrm{FEV}_{1} \\
(\% \text {, mean } \pm \text { SD) }\end{array}$ & $20.1 \pm 6.4$ & $20.3 \pm 6.1$ & .94 \\
\hline \multicolumn{4}{|l|}{ Ischemia time (min, mean $\pm S D$ ) } \\
\hline $\begin{array}{l}\text { For 1st allograft } \\
\text { For 2nd allograft }\end{array}$ & $\begin{array}{l}205.1 \pm 50.1 \\
322.3 \pm 59.4\end{array}$ & $\begin{array}{l}254.7 \pm 55.2 \\
384.6 \pm 79.3\end{array}$ & $\begin{array}{r}0.11^{*} \\
.4^{*}\end{array}$ \\
\hline
\end{tabular}

$P$ values were calculated by analysis of variance.

${ }^{*}$ Adjusted for surgeons as covariates in the analyses.

the official radiologic report in the following manner: 0, normal; 1 , perihilar infiltrate or infiltrate localized to a limited lung field; 2, mild diffuse infiltrate or edema; 3 , moderate diffuse infiltrate; and 4 , severe diffuse infiltrate. The difference in infiltrate scores between the first and second allograft implanted was used as one assessment of whether CPB protects the first allograft implanted from hyperperfusion injury. Furthermore, the number of patients, in whom the first allograft infiltrate score was at least 2 grades higher than the second allograft score was determined. Arterial blood gas (ABG) values obtained at approximately 1, 24, and 48 hours after transplantation were reviewed to calculate the $\mathrm{PaO}_{2}$ to fraction inspired oxygen ratio $\left(\mathrm{PaO}_{2} / \mathrm{FIO}_{2}\right)$. The 1-hour $\mathrm{ABG}$ reading was the first $\mathrm{ABG}$ assessment obtained on arrival in the intensive care unit (ICU). Pulmonary function tests (percentages of predicted forced vital capacity and $\mathrm{FEV}_{1}$ ) at 1 year after transplantation were reviewed to assess the intermediate-term allograft function.

\section{Clinical Outcome}

ICU stay and hospital stay in days after transplantation were reviewed. The change in postoperative renal function was analyzed by reviewing the baseline and the peak serum creatinine levels attained during the hospital course. The mean increase in serum creatinine level (peak minus baseline) was calculated. Patient records were also reviewed for bronchial anastomotic complications and the prevalence of bronchiolitis obliterans syndrome (BOS), as defined by the International Society for Heart and Lung Transplantation. ${ }^{12}$ Transfusion requirements in terms of packed red blood cells, fresh-frozen plasma, and platelets were compared by reviewing blood bank records. The 30-day mortality and 1-year and 3-year survivals were compared to examine the short- and intermediate-term clinical outcomes.

\section{Statistical Analysis}

Inspection of each outcome measure was conducted to determine the nature of its distribution. The choice of statistical model was dependent on each outcome distribution. For measures that appeared to be normally distributed or through a transformation were normally distributed, analysis of variance was used. For measures for which transformation did not lead to a normal distribution, alternate statistical models were implemented. Analysis of variance was used to compare the age of the recipients, preoperative and postoperative pulmonary function tests, $\mathrm{PaO}_{2} / \mathrm{FIO}_{2}$ ratio, increase in serum creatinine level, and ischemic times of the allografts. To adjust for surgeon variability, the surgeons were factored as covariates (analysis of covariance) only when analyzing ischemic times of the allografts. The cumulative logit model was used to evaluate the chest radiographic infiltrate scores. Because of their count nature, Poisson regression was used to evaluate ICU and hospital stays, mechanical ventilation requirement, and transfusion requirements (packed red blood cells, fresh-frozen plasma, platelets). Logistic regression was used to evaluate the 30-day mortality and 1- and 3-year survivals. In addition, Kaplan-Meier analysis (Wilcoxon test) was performed on the entire population and a subset of the non-CPB control patients (with transplantation between 1996 and 2000) to address the difference in the operation dates between the two groups for the survival analysis and to account for the heavy censoring. The Fisher exact test was used for small samples (30-day mortality). Bronchial anastomotic complication rate and the prevalence of BOS were also analyzed with the Fisher exact test.

\section{Results \\ Recipient Demographic Data}

The mean duration of CPB was $218.3 \pm 75.4$ minutes. As stated previously, the decision to use CPB to provide controlled pulmonary reperfusion pressure and flow to the newly implanted allografts was made before recipient pneumonectomy. The recipient demographic characteristics are shown in Table 1. No significant differences in recipients' age $(P=.13)$, sex $(P=.93)$, preoperative pulmonary function tests (percentage of predicted forced vital capacity $P=.94$, percentage of predicted $\left.\mathrm{FEV}_{1} P=.94\right)$, and allograft ischemic time (first lung $P=.11$, second lung $P=$ .40) were seen. These findings confirmed the comparability of the populations of patients for this study.

\section{Postoperative Allograft Function}

There was no significant difference in postoperative mechanical ventilation requirement (median duration of intubation in days) between the CPB group (1 day) and the non-CPB group (1 day, $P=.76$; Figure 1 ). The mean 


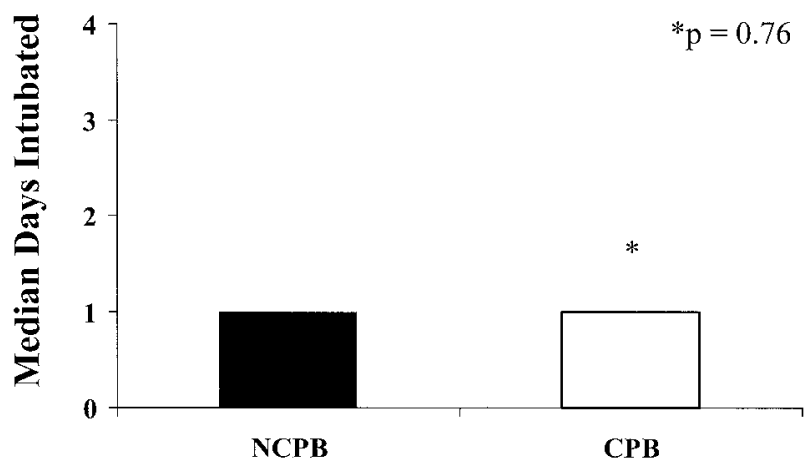

Figure 1. Requirement for mechanical ventilation after lung transplantation in CPB and non-CPB (NCPB) groups. Asterisk indicates $P=.76$.

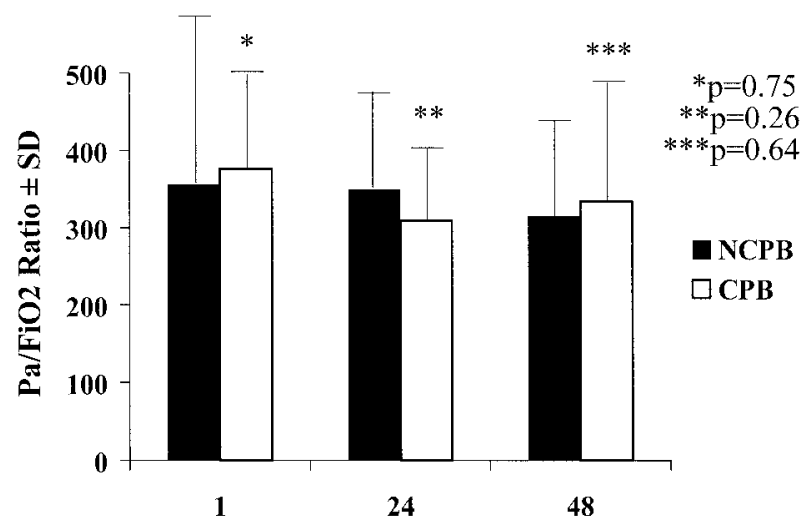

Time After Transplantation (Hours)

Figure 2. Postoperative $\mathrm{PaO}_{2} / \mathrm{FiO}_{2}$ ratio in CPB group (white bars) and non-CPB group (black bars). Bar heights represent mean; error bars represent SD. Asterisk indicates $P=.75$; double asterisk indicates $\boldsymbol{P}=.26$; triple asterisk indicates $\boldsymbol{P}=.64$.

differences in chest radiographic infiltrate scores between the first and second lungs implanted were compared at three time points after transplantation (1,24, and 48 hours). At 1 hour after transplantation, the mean $( \pm \mathrm{SD})$ differences in infiltrate score between the first and second allografts were $0.4 \pm 0.5$ in the CPB group and $0.2 \pm 0.8(P=.19)$ in the non-CPB group. At 24 hours, the differences in infiltrate scores were $0.3 \pm 0.5$ and $0.2 \pm 0.6$ in the $\mathrm{CPB}$ and non-CPB groups, respectively $(P=.65)$. At 48 hours, the differences in infiltrate score were $0.1 \pm 0.4$ in the $\mathrm{CPB}$ group and $0.1 \pm 0.4$ in the non-CPB group $(P=.99)$. None of the 14 patients in the $\mathrm{CPB}$ group had an infiltrate score in the first lung implanted that was 2 grades or more higher than in the second lung implanted at 1 hour, whereas 3 patients in the non-CPB group $(\mathrm{n}=36)$ had an infiltrate score in the first lung implanted that was 2 grades or more higher than that in the second lung implanted at 1 hour. This trend was not statistically significant $(P=.55)$. The ABG

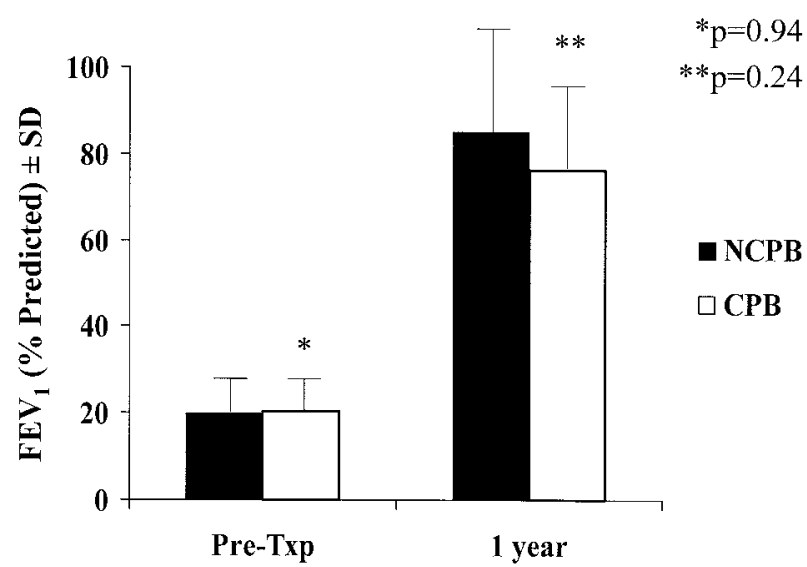

Figure 3. Pulmonary function (forced expiratory volume in 1 second, FEV $_{1}$ ) before (Pre-Txp) and 1 year after lung transplantation in CPB group (white bars) and non-CPB group (black bars). Bar heights represent mean; error bars represent SD. Asterisk indicates $\boldsymbol{P}=.94$; double asterisk indicates $\boldsymbol{P}=.24$.

values were examined to calculate the $\mathrm{PaO}_{2} / \mathrm{FIO}_{2}$ ratios after transplantation at intervals of 1, 24, and 48 hours (Figure 2). Immediately on arrival in the ICU, the mean $\mathrm{PaO}_{2} / \mathrm{FIO}_{2}$ ratio in the CPB group was $376.6 \pm 123$ and that in the non-CPB group was $357.0 \pm 218(P=.75)$. These were recorded as the 1-hour $\mathrm{PaO}_{2} / \mathrm{FIO}_{2}$ ratios, because the $\mathrm{ABG}$ values were in all cases obtained at least 1 hour after reperfusion of the allografts. At 24 hours, the $\mathrm{PaO}_{2} / \mathrm{FIO}_{2}$ ratios were $309.9 \pm 92$ and $350.6 \pm 122$ in the CPB and non-CPB groups, respectively $(P=.26)$. At 48 hours, the $\mathrm{PaO}_{2} / \mathrm{FIO}_{2}$ ratio in the $\mathrm{CPB}$ group was $335.0 \pm 144$ and that in the non-CPB group was $316.2 \pm 120(P=.64)$. Because this was not a prospective study, ABG values were not obtained at exactly 24 and 48 hours. The closest ABG determination to the reported interval was therefore used. At 1 year after transplantation, pulmonary function tests were performed to assess the intermediate-term allograft function (Figure 3). There were no significant differences between the two groups in the percentage of expected $\mathrm{FEV}_{1}$ (CPB $76.1 \% \pm 17.0 \%$, non-CPB $85.3 \% \pm 21.7 \%, P=.24)$.

\section{Clinical Outcome}

The median stay in the ICU was the same for the CPB and non-CPB groups (4 days, $P=.44$, Figure 4 ). Similarly, there was no difference in the median duration of hospital stay (CPB 15.5 days, non-CPB 16 days, $P=.74$, Figure 4 ). The mean perioperative increases in serum creatinine level (peak minus baseline level) were $1.4 \pm 1.9 \mathrm{mg} / \mathrm{dL}$ and $0.9 \pm 1.0 \mathrm{mg} / \mathrm{dL}(P=.33)$ in the CPB and non-CPB groups, respectively. Bronchial anastomotic stenosis requiring stent placement or dilatation occurred in 1 of 14 patients $(7.1 \%)$ in the CPB group and in 4 of 36 patients $(11.1 \%)$ in the non-CPB group $(P>.999)$. Bronchial dehiscence did 


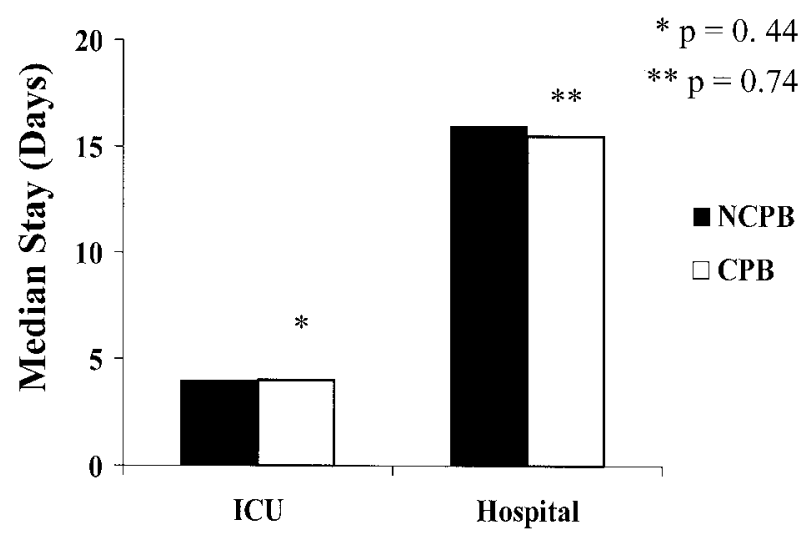

Figure 4. Lengths of ICU and hospital stays after lung transplantation in CPB group (white bars) and non-CPB group (black bars). Asterisk indicates $P=.44$; double asterisk indicates $P=.74$.

not occur in either group. None of the 14 patients in the CPB group showed clinical evidence of BOS, whereas 13 of 36 patients $(36.1 \%)$ in the non-CPB group had clinical evidence of decline in pulmonary function consistent with BOS $(P=.01)$. To correct for potential differences in the time allowed for development of BOS, a subset $(\mathrm{n}=13)$ of patients in the non-CPB group who underwent transplantation between 1996 and 2000 was analyzed. The prevalence of BOS in this group was 4 of $13(30.8 \%)$, which was significantly different from the $0 \%$ prevalence of BOS in the 14 patients in the CPB group $(P=.047)$. However, blood product requirements were higher in the CPB group (packed red blood cells $6.5 \pm 4.1$ units vs $2.1 \pm 1.7$ units, $P<.001$, platelets $1.4 \pm 1.4$ units vs $0.1 \pm 0.3$ units, $P<.001$, fresh-frozen plasma $11.0 \pm 7.7$ units vs $0.3 \pm 0.8$ units, $P<.001$; Figure 5).

\section{Survival}

The effects of CPB on short- and intermediate-term survival were examined (Table 2). When the Fisher exact test and logistic regression were used to analyze the entire study period, the 30-day mortalities were similar in the CPB and non-CPB groups ( $7.1 \%$ vs $8.3 \%, P=1.0)$. Similarly, intermediate-term survival data were also not significantly different between the two groups. The 1-year and 3-year survivals in the CPB group (85.7\% and $64.3 \%$, respectively) were comparable to those in the non-CPB group (80.1\%, $P=.66$, and $58.3 \%, P=.70$ ). A Kaplan-Meier analysis (Wilcoxon test) of the entire study period also demonstrated that the median survival times were not significantly different between the CPB group (1407 days) and the non-CPB group (2591 days, $P=.86$ ). A Kaplan-Meier analysis (Wilcoxon test) of patients who underwent transplantation between 1996 and 2000 also demonstrated that there was no difference in survival between the $\mathrm{CPB}$ and non-CPB groups $(P=.73)$. This subset analysis was performed to

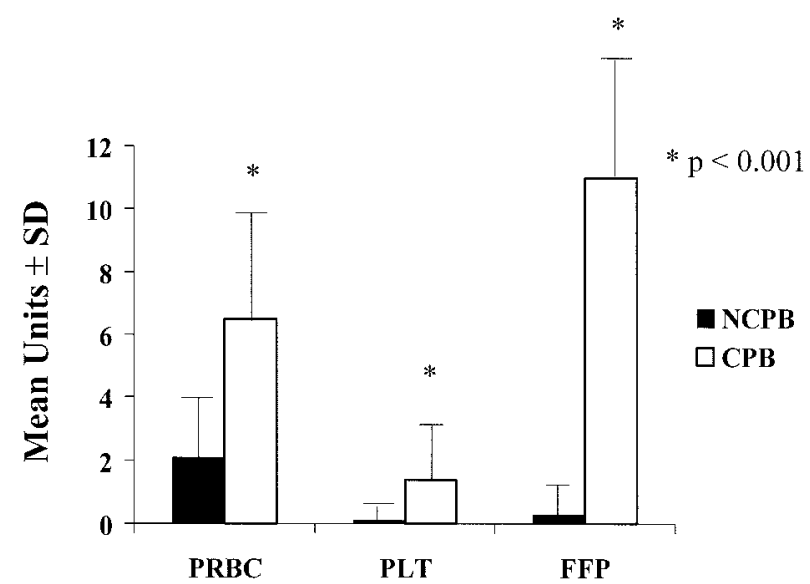

Figure 5. Postoperative blood product requirements in CPB group (white bars) and non-CPB group (black bars). Bar heights represent mean; error bars represent SD. Asterisk indicates $P<.001$. PRBC, Packed red blood cells; PLT, platelets; FFP, fresh-frozen plasma.

TABLE 2. Survivals

\begin{tabular}{lcrr}
\hline & Non-CPB & CPB & P value \\
\hline 30-d mortality & 8.3 & 7.1 & $>.999^{*}$ \\
1-y survival & 80.1 & 85.7 & $.66 \dagger$ \\
3-y survival & 58.3 & 64.3 & $.70 \dagger$
\end{tabular}

*By 2-sided Fisher exact test.

tBy logistic regression analysis.

address the limitations of the Kaplan-Meier method, which biases in favor of patients with longer follow-ups in the setting of a small sample size and significant censoring of data. This subset of patients who underwent transplantation between 1996 and 2000 was also used to address the learning curve in our experience in lung transplantation. In this subset of patients, the Fisher exact test and logistic regression model again demonstrated no difference in the 30-day mortality (CPB $7.1 \%$ vs non-CPB $15.3 \%, P=.60$ ) and the 1 -year survival (CPB $85.7 \%$ vs non-CPB $69.2 \%, P=.30$ ). The 3 -year survival was $64.3 \%$ in the CPB group and $23.1 \%$ in the non-CPB group $(P=.03)$. The causes of death are listed in Table 3.

\section{Discussion}

The introduction of bilateral sequential lung transplantation has provided surgeons the ability to perform double-lung transplantation without the use of CPB. ${ }^{1}$ Since inception of the procedure, the use of $\mathrm{CPB}$ in lung transplantation has remained a much debated topic. Experimental and clinical investigators have reached differing conclusions regarding the effects of CPB in lung transplantation. Our review of the current literature found that the retrospective clinical studies to date are weakened by inclusion of patients with different 
TABLE 3. Causes of death

\begin{tabular}{lcc}
\hline & Non-CPB & CPB \\
\hline BOS & 3 & 0 \\
Primary graft failure & 2 & 1 \\
Posttransplantation lymphoproliferative disease & 1 & 1 \\
Pneumonia & 1 & 1 \\
Myocardial infarction & 1 & 0 \\
Colitis or perforation & 2 & 0 \\
Chronic renal failure and congestive heart & 0 & 1 \\
$\quad$ failure & & \\
Lung malignancy & 1 & 0 \\
Guillain-Barré syndrome & 1 & 0 \\
\hline
\end{tabular}

Data represent numbers of patients.

diagnoses, both single- and double-lung transplants, and both elective and emergency use of CPB. These confounding variables prevent clear assessment of whether $\mathrm{CPB}$ is in fact deleterious. Patients with different diagnoses represent a heterogeneous population with contrasting medical conditions and background. In the setting of single-lung transplantation, the remaining native lung can have a significant effect on short-term and long-term posttransplantation pulmonary function. Circumstances involving the use of $\mathrm{CPB}$ can also have effects on clinical outcome. Emergency use of $\mathrm{CPB}$ resulting from hemodynamic instability or oxygen desaturation may be a surrogate for patients with poor pulmonary reserve, thus introducing patient selection bias. Moreover, borderline or frank shock before institution of CPB clearly has multisystemic adverse effects on organ function. We believe that the current opinion in the literature regarding the deleterious effect of $\mathrm{CPB}$ in lung transplantation may not be entirely warranted. The goal of this study was to determine whether CPB is deleterious by conducting a study devoid of the bias potentially introduced by these factors. To achieve this, our study examined only patients with diagnosis of COPD undergoing bilateral sequential lung transplantation with or without elective CPB. Unlike many centers, we have a sizable number of patients with COPD and emphysema undergoing bilateral sequential lung transplantation because of a large number of younger patients referred with $\alpha_{1}$-antitrypsin disease. We have previously reported excellent results with this approach. ${ }^{13,14}$

Our study found that CPB was not associated with any deleterious effects on lung function, although we recognize that the small size of the study limits our ability to detect subtle differences. In terms of allograft function, the CPB group was not significantly different in duration of intubation, $\mathrm{PaO}_{2} / \mathrm{FIO}_{2}$ ratio, and 1-year pulmonary function test results. We also compared the chest radiographic infiltrate scores between the first and second lung implanted, recognizing that this is an unproven marker of asymmetric injury. The difference between the first and the second lung implanted infiltrate scores was not significantly different be- tween the CPB group the non-CPB group. This assessment was not particularly sensitive, however, because our method of chest radiographic assessment was to assign grades on the basis of the official radiology report. It is therefore not surprising that mean infiltrate scores were not sensitive enough to demonstrate a difference. However, when we looked at the percentage of patients with a marked difference in infiltrate score between the two lungs (at least a 2 -grade difference), we found that 3 of 36 patients in the non-CPB group had radiologic evidence of significant "first lung" injury. In contrast, none of the 14 patients in the CPB group had evidence of similarly severe injury. Although this trend was not statistically significant, it shows that CPB did not have a major deleterious effect and even suggests that CPB may be beneficial. To prove that hypothesis would clearly require a much larger study involving blinded, prospective scoring of chest radiographs. In terms of clinical outcome, the CPB group also did not exhibit significant differences in ICU stay, hospital stay, renal function, bronchial anastomotic complications, 30-day mortality, and 1 -year and 3-year survivals. However, we did find that the prevalence of BOS was lower in the CPB group (0\%) than in the non-CPB group (36.1\%). Although this finding was statistically significant, we suspect that it may not reflect a true biologic phenomenon, because it is well established that the prevalence of BOS among heart-lung transplant recipients, who all require $\mathrm{CPB}$, is no lower than in lung transplant recipients, some of whom do not undergo CPB. We did find a significant increase in blood transfusion requirements in the CPB group.

Approximately two thirds of the transplants performed in the non-CPB group occurred between 1993 and 1996. This is in contrast to the CPB group, in which all transplantation procedures were performed from 1996 to 2000. This raises the possibility that our results merely reflect a learning curve. Moreover, the differences in the starting dates between the two groups introduce heavily censored data into the Kaplan-Meier analysis. This is particularly relevant because our sample size was small. To address these issues, we analyzed a subset of patients who underwent lung transplantation between the period of 1996 to 2000 by means of logistic regression analysis. We found no statistically significant differences between the CPB $(n=14)$ and the non-CPB $(n=13)$ groups in the same parameters analyzed for the entire study period. However, we did see a better 3 -year survival $(64.3 \%)$ in the CPB group than in the non-CPB group $(23.1 \%, P=.03)$. The impact of the learning curve for transplantation on patient clinical outcome is a valid concern, but the analysis of the subset of patients in the later group (1996 to 2000) suggested that it did not have a significant effect in our experience. To address the issue of heavily censored data, Kaplan-Meier analysis with the Wilcoxon test was performed. The analysis of the entire popu- 
lation as well as the subset of contemporaneous non-CPB control patients showed survival to be equivalent between the CPB and non-CPB groups. Survival appeared to be similar between the two groups once heavy censoring was accounted for, suggesting the significance of censoring in this sample size. The efficiency of the results from the logistic regression analysis was not impaired by the heavy censoring, further supporting its use as the optimal method for comparing the 30-day, 1-year, and 3-year survivals.

The theory that $\mathrm{CPB}$ may control reperfusion pressure and flow during implantation of second lung, thereby reducing overall ischemia-reperfusion injury to the first implanted allograft, has stimulated much debate and investigation. Without CPB, the diversion of the entire cardiac output to the first implanted lung can result in pulmonary overcirculation, which often translates into allograft dysfunction. In cardiac surgery as well as orthotopic heart transplantation, the benefits of controlled reperfusion with the use of CPB are well established both experimentally ${ }^{15}$ and clinically. ${ }^{16,17}$ The concerns of sudden reperfusion injury appear valid for lung transplantation. Experimental data have shown that increased flow and pressure may lead to lung dysfunction. In a canine model, Fullerton and associates $^{3}$ demonstrated that chronically increased pulmonary blood flow after creating a 3:1 left-to-right shunt resulted in dysfunctional pulmonary vasorelaxation. In an ex vivo rabbit model, DeLima and associates ${ }^{2}$ demonstrated that rabbit lungs receiving high-flow reperfusion resulted in an increase in pulmonary vascular resistance, a decrease in dynamic airway compliance, and an increased wet/dry weight ratio. Furthermore, experimental and clinical studies have demonstrated the benefits of controlled reperfusion in lung transplantation. In an ex vivo rat lung model, Hopkinson and associates ${ }^{4}$ demonstrated that controlled pressure reperfusion of pulmonary grafts resulted in improved pulmonary function. In an in vivo porcine lung transplantation model, Halldorsson and associates ${ }^{18-20}$ demonstrated that reperfusion injury was avoided by controlling the composition (modified solution, leukocyte filter) and the conditions (pressure and rate) of the reperfusate. Recently, Lick and associates ${ }^{21}$ reported a clinical series of 5 patients undergoing lung transplant procedures with a modification of the technique described by Halldorsson and associates. ${ }^{18}$

Controlled reperfusion was achieved by passing the reperfusate through a leukocyte filter and into the transplant pulmonary artery at a controlled rate $(200 \mathrm{~mL} / \mathrm{min})$ and pressure $(<20 \mathrm{~mm} \mathrm{Hg})$. The investigators reported excellent functional results in these patients compared with historical control patients at their institution. ${ }^{21}$

In addition to our review, other clinical studies have also added evidence that CPB is not deleterious to clinical outcome after lung transplantation. In a retrospective review of 162 patients, Triantafillou and associates ${ }^{11}$ found no differ- ence between the $\mathrm{CPB}$ and non-CPB groups in terms of time until extubation, time spent in the ICU, and time required to reach a $\mathrm{Po}_{2}$ on room air greater than $60 \mathrm{~mm} \mathrm{Hg}$. In a review of the Cleveland Clinic experience, Hlozek and associates ${ }^{10}$ found no difference between the CPB and non-CPB groups in terms of patient survival, initial $\mathrm{PaO}_{2} / \mathrm{FIO}_{2}$, and length of ICU stay. However, they did find a significant difference in the duration of intubation and blood transfusion requirement.

Opponents of $\mathrm{CPB}$ argue that $\mathrm{CPB}$ elicits a "wholebody" inflammatory response that may be detrimental to the pulmonary allograft. It has been well documented that $\mathrm{CPB}$ activates leukocytes ${ }^{22,23}$ and the complement pathway ${ }^{24}$ and increases the release of cytokines and interleukins. ${ }^{25,26}$ The introduction of activated blood components may result in acute lung injury. Fullerton and associates ${ }^{7}$ demonstrated in a canine lung transplantation model that CPB exaggerates pulmonary vasomotor dysfunction in the transplanted lung, which results in a higher pulmonary vascular resistance. Clinical studies have also suggested the CPB may have a deleterious effect on patient morbidity and mortality. In a retrospective review of 100 patients undergoing lung transplantation, Aeba and associates ${ }^{9}$ found that patients in the CPB group had significantly worse arterial-alveolar $\mathrm{PO}_{2}$ ratio, more severe pulmonary infiltrates on chest radiographs, longer duration of intubation, and worse survival at 1 month after operation than did the non-CPB group. Similarly, in a retrospective review of 94 double-lung transplantations, Gammie and associates ${ }^{8}$ found that CPB was associated with more perioperative transfusion requirements, lower arterial-alveolar $\mathrm{Po}_{2}$ ratio, more severe pulmonary infiltrates, and longer duration of intubation. However, the 30 -day mortality and the 1 -year survival were not significantly different between groups.

Even though our data suggest that CPB has no deleterious effect, we understand that our study has some limitations. First, we cannot conclude that $\mathrm{CPB}$ is beneficial on the basis of our data. However, the goal of this study was to test the hypothesis that CPB has no deleterious effects on graft function and therefore clinical outcome in lung transplantation, once confounding factors are eliminated. We believe that controlled reperfusion is an important concept and hope that this study will raise significant questions about the adverse effects of CPB in lung transplantation. We believe that prospective, randomized studies to assess the role of controlled reperfusion in minimizing ischemic-reperfusion injury are warranted. Second, for a comparison of a homogeneous population undergoing the same procedure, the number of patients in the study was small. We realize that a type II error, or failure to reject a null hypothesis when it is false, is possible in this study. Our study does not have the power to definitively rule out any deleterious effect of $\mathrm{CPB}$. We have performed power calculations for this 
study's findings, and for an $\alpha$ of .05 and a power of $80 \%$, we would have needed 26 patients per group to detect a large effect size, 65 patients per group to detect a medium effect size, and 390 patients per group to detect a small effect size. Third, we realize that this is a retrospective review, and the decision to use CPB electively is biased by the individual surgeon's preference. Finally, patients were not randomly assigned to study the effect of CPB. As previously discussed, a prospective, randomized study with a significant sample size would be required to definitely conclude that CPB does not deleteriously affect lung function or clinical outcome.

The technical aspects of lung transplantation are challenging and continue to evolve at our institution. Our current practice is to use $\mathrm{CPB}$ in the setting of pulmonary hypertension (mean $\mathrm{PaO}_{2} 40 \mathrm{~mm} \mathrm{Hg}$ ) on maximal support $\left(\mathrm{PCO}_{2} 25-30 \mathrm{~mm} \mathrm{Hg}\right.$, inhaled nitric oxide at $40 \mathrm{ppm}$, isoproterenol at the maximum tolerable dose keeping heart rate $<110$ beats/min, and alprostadil at the maximum tolerable dose keeping mean arterial pressure $60 \mathrm{~mm} \mathrm{Hg}$ ). Our practice in the setting of bilateral sequential lung transplantation in the absence of pulmonary hypertension continues to evolve. Currently we favor routine elective use of CPB during the implantation of the second pulmonary allograft to control perfusion pressure in the first lung, to eliminate hemodynamic instability or hypoxia during the procedure, and to aid in exposure of the left hilar structures. Although there is evidence suggesting that the use of heparin-bonded circuits and leukocyte filtration may provide additional benefit, we do not currently use this approach in our program. Whether controlled reperfusion is protective against ischemia-reperfusion injury is still unknown. However, our study suggests that there is minimal deleterious effect of CPB on lung transplantation used electively in the specific setting of bilateral sequential lung transplantation for patients with COPD. Even though CPB resulted in a higher transfusion requirement, it did not adversely affect allograft function and clinical outcome. Although we are not advocating routine use of CPB for either single- or double-lung transplantation procedures, we hope that this study will remove some of the unwarranted fear of the use of CPB in lung transplantation for COPD.

\section{References}

1. Kaiser LR, Pasque MK, Trulock EP, Low DE, Dresler CM, Cooper JD. Bilateral sequential lung transplantation: the procedure of choice for double-lung replacement. Ann Thorac Surg. 1991;52:438-45.

2. DeLima NF, Binns OA, Buchanan SA, Mauney MC, Cope JT, Shockey KS, et al. Euro-Collins solution exacerbates lung injury in the setting of high-flow reperfusion. J Thorac Cardiovasc Surg. 1996;112: $111-6$.

3. Fullerton DA, Mitchell MB, Jones DN, Maki A, McIntyre RC. Pulmonary vasomotor dysfunction is produced with chronically high pulmonary blood flow. J Thorac Cardiovasc Surg. 1996;111:190-7.

4. Hopkinson DN, Bhabra MS, Odom NJ, Bridgewater BJ, Van Doorn CA, Hooper TL. Controlled pressure reperfusion of rat pulmonary grafts yields improved function after twenty-four-hours' cold storage in University of Wisconsin solution. J Heart Lung Transplant. 1996; 15:283-90.

5. Wan S, LeClerc JL, Vincent JL. Inflammatory response to cardiopulmonary bypass: mechanisms involved and possible therapeutic strategies. Chest. 1997;112:676-92.

6. Francalancia NA, Aeba R, Yousem SA, Griffith BP, Marrone GC. Deleterious effects of cardiopulmonary bypass on early graft function after single lung allotransplantation: evaluation of a heparin-coated bypass circuit. J Heart Lung Transplant. 1994;13:498-507.

7. Fullerton DA, McIntyre RC Jr, Mitchell MB, Campbell DN, Grover FL. Lung transplantation with cardiopulmonary bypass exaggerates pulmonary vasomotor dysfunction in the transplanted lung. $J$ Thorac Cardiovasc Surg. 1995;109:212-6.

8. Gammie JS, Cheul Lee J, Pham SM, Keenan RJ, Weyant RJ, Hattler $\mathrm{BG}$, et al. Cardiopulmonary bypass is associated with early allograft dysfunction but not death after double-lung transplantation. $J$ Thorac Cardiovasc Surg. 1998;115:990-7.

9. Aeba R, Griffith BP, Kormos RL, Armitage JM, Gasior TA, Fuhrman $\mathrm{CR}$, et al. Effect of cardiopulmonary bypass on early graft dysfunction in clinical lung transplantation. Ann Thorac Surg. 1994:57:715-22.

10. Hlozek CC, Smedira NG, Kirby TJ, Patel AN, Perl M. Cardiopulmonary bypass (CPB) for lung transplantation. Perfusion. 1997;12:10712.

11. Triantafillou AN, Pasque MK, Huddleston CB, Pond CG, Cerza RF, Forstot RM, et al. Predictors, frequency, and indications for cardiopulmonary bypass during lung transplantation in adults. Ann Thorac Surg. 1994;57:1248-51.

12. Cooper JD, Billingham M, Egan T, Hertz MI, Higenbottam T, Lynch $\mathrm{J}$, et al. A working formulation for the standardization of nomenclature and for clinical staging of chronic dysfunction in lung allografts. International Society for Heart and Lung Transplantation. J Heart Lung Transplant. 1993;12:713-6.

13. Bavaria JE, Kotloff R, Palevsky H, Rosengard B, Roberts JR, Wahl $\mathrm{PM}$, et al. Bilateral versus single lung transplantation for chronic obstructive pulmonary disease. J Thorac Cardiovasc Surg. 1997;113: 520-7.

14. Pochettino A, Kotloff RM, Rosengard BR, Arcasoy SM, Blumenthal NP, Kaiser LR, et al. Bilateral versus single lung transplantation for chronic obstructive pulmonary disease: intermediate-term results. Ann Thorac Surg. 2000;70:1813-1.

15. Okamoto F, Allen BS, Buckberg GD, Bugyi H, Leaf J. Reperfusion conditions: importance of ensuring gentle versus sudden reperfusion during relief of coronary occlusion. J Thorac Cardiovasc Surg. 1986; 92(3 Pt 2):613-20.

16. Beyersdorf F, Sarai K, Maul FD, Wendt T, Satter P. Immediate functional benefits after controlled reperfusion during surgical revascularization for acute coronary occlusion. J Thorac Cardiovasc Surg. 1991;102:856-66.

17. Fontan F, Madonna F, Naftel DC, Kirklin JW, Blackstone EH, Digerness S. Modifying myocardial management in cardiac surgery: a randomized trial. Eur J Cardiothorac Surg. 1992;6:127-36.

18. Halldorsson A, Kronon M, Allen BS, Bolling KS, Wang T, Rahman $\mathrm{S}$, et al. Controlled reperfusion after lung ischemia: implications for improved function after lung transplantation. $J$ Thorac Cardiovasc Surg. 1998;115:415-24.

19. Halldorsson AO, Kronon M, Allen BS, Rahman S, Wang T, Layland M, et al. Controlled reperfusion prevents pulmonary injury after 24 hours of lung preservation. Ann Thorac Surg. 1998;66:877-84.

20. Halldorsson AO, Kronon MT, Allen BS, Rahman S, Wang T. Lowering reperfusion pressure reduces the injury after pulmonary ischemia. Ann Thorac Surg. 2000;69:198-203.

21. Lick SD, Brown PS Jr, Kurusz M, Vertrees RA, McQuitty CK, Johnston WE. Technique of controlled reperfusion of the transplanted lung in humans. Ann Thorac Surg. 2000;69:910-2.

22. Gu YJ, van Oeveren W, Boonstra PW, de Haan J, Wildevuur CR. Leukocyte activation with increased expression of CR3 receptors during cardiopulmonary bypass. Ann Thorac Surg. 1992;53:839-43.

23. Kharazmi A, Andersen LW, Baek L, Valerius NH, Laub M, Rasmussen JP. Endotoxemia and enhanced generation of oxygen radicals by 
neutrophils from patients undergoing cardiopulmonary bypass. $J$ Thorac Cardiovasc Surg. 1989;98:381-5.

24. Kirklin JK, Westaby S, Blackstone EH, Kirklin JW, Chenoweth DE, Pacifico AD. Complement and the damaging effects of cardiopulmonary bypass. J Thorac Cardiovasc Surg. 1983;86:845-57.
25. Downing SW, Edmunds LH. Release of vasoactive substances during cardiopulmonary bypass. Ann Thorac Surg. 1992;54:1236-43.

26. Butler J, Chong GL, Baigrie RJ, Pillai R, Westaby S, Rocker GM. Cytokine responses to cardiopulmonary bypass with membrane and bubble oxygenation. Ann Thorac Surg. 1992;53:833-8.

\section{JTCVS On-Line Manuscript Submission and Review}

Please visit http://www.editorialmanager.com/jtcvs/

Effective September 15, 2001, authors and reviewers may submit manuscripts and reviews electronically via Editorial Manager, our new Web-based system with full electronic submission, review, and status update capabilities.

As we move from paper to electronic submissions, the Editorial Office will make proxy submissions of all manuscripts accompanied by a diskette containing the electronic files of the text, tables, and figures. Editors, authors, and reviewers will receive automatic e-mails when significant events occur.

We strongly encourage all authors and reviewers to use Editorial Manager. Although we will continue to accommodate the submission of paper manuscripts for some months, our goal is to be completely electronic within 9 to 12 months.

All individuals currently in our database for whom we have e-mail addresses will receive via e-mail a system-assigned username and password that can be used to log in to the system without prior registration. All those not receiving the e-mail must register the first time they use the system.

As with any broad systemic change, the conversion to the new system will take some time to complete. We ask your patience as we replace our in-office database with the new system. We also encourage you to take advantage of the speed and efficiency that the new system will provide for us all: editor, author, reviewer, and publisher. 